\title{
A Flexible Bayesian Approach to Bias Correction of Radar-Derived Precipitation Estimates over Complex Terrain: Model Design and Initial Verification
}

\author{
HAONAN CHEN \\ Physical Sciences Division, NOAA/Earth System Research Laboratory, Boulder, and Cooperative Institute for \\ Research in the Atmosphere, Fort Collins, Colorado \\ RoB CIFELLI \\ Physical Sciences Division, NOAA/Earth System Research Laboratory, Boulder, Colorado \\ V. CHANDRASEKAR AND YingZHAO MA \\ Colorado State University, Fort Collins, Colorado
}

(Manuscript received 24 June 2019, in final form 15 October 2019)

\begin{abstract}
This study develops a flexible Bayesian technique to quantify uncertainties associated with the operational Weather Surveillance Radar-1988 Doppler (WSR-88D) quantitative precipitation estimation (QPE) products over complex terrain. Radar-only rainfall estimates and rain gauge observations over the Russian River watershed in Northern California are utilized to demonstrate this new bias correction approach. Conventional mean field bias (MFB) and local bias (LB) correction methods are also implemented for comparison purposes. Results show that the proposed Bayesian technique outperforms the conventional MFB and LB correction approaches. The radar QPE performance is dramatically improved after the Bayesian-based bias correction: the root-mean-square error is reduced from 4.2 to $1.71 \mathrm{~mm}$, the normalized mean absolute error is reduced from $64.5 \%$ to $24.2 \%$, and the correlation with gauge measurements increases from 0.11 to 0.74 . In addition, the terrain impact on radar QPE bias correction performance is investigated. After incorporating the terrain elevation information in the Bayesian framework, the QPE performance is further enhanced. Overall, the QPE performance scores after including the terrain information are improved about $10 \%$ relative to those only based on rainfall intensity values.
\end{abstract}

\section{Introduction}

Obtaining accurate quantitative precipitation estimation (QPE) using rain gauges and/or remote sensing instruments such as radar and satellite is a problem of continuing interest to meteorologists and hydrologists. There are a number of advantages of using radar since radar can observe precipitation over a wide area in a relatively short period of time (Doviak and Zrnić 1993; Bringi and Chandrasekar 2001; Zhang et al. 2011; Chen et al. 2019a). However, it is nontrivial to construct a robust functional relation between the radar measurements aloft and rainfall rate on the ground (Fulton et al. 1998; Anagnostou et al. 1999; Krajewski and Smith 2002; Chen et al.2017). There are many issues in the process of

Corresponding author: Haonan Chen, haonan.chen@noaa.gov converting radar observations into reliable QPE products that can be used for subsequent applications. These issues, if not handled well, can introduce significant errors and uncertainty in the derived surface precipitation estimates and hydrologic forecasts.

In general, the associated errors in radar QPE can be classified into four categories: 1) radar system calibration or measurement error; 2) data quality control due to wave propagation in complex environments; 3 ) parameterization error in radar rainfall relations; 4) mismatch between radar estimates aloft and surface rainfall at the ground. The first category is commonly referred to the accuracy of radar system components (i.e., transmitter, receiver, etc.), and the measurement accuracy of reflectivity or other polarimetric radar moments that can be used to derive QPE. The data quality control and environmental factors include clutter filtering, nonmeteorological 
echo suppression, and anomalous radar beam propagation. Beam blockage resulting from part of the radar beam intercepting obstacles such as buildings and mountains is another important environmental factor that affects QPE. This is manifest especially in complex topography such as the western United States, where the operational weather radars are deployed in mountainous terrain (Maddox et al. 2002; Houze 2012; Willie et al. 2017; Cifelli et al. 2018; Bytheway et al. 2019). In addition, substantial variability in the vertical profile of reflectivity (VPR) occurs as a result of precipitation growth, evaporation, and melting of ice particles and snowflakes (Bellon et al. 2005; Zhang and Qi 2010; Gou et al. 2018). Such variations indicate that there can be large differences between actual radar measurements in the atmosphere and ideal observations occurring at the ground. VPR correction is often required in the data quality control process in order to compensate such variations and derive surface level precipitation estimates. On the other hand, the radar beam is likely to either partially or completely overshoot shallow precipitation at long ranges, resulting in either underestimation of the precipitation rate or complete failure to detect precipitation respectively. The third category of error arises from the assumption on the physical model of raindrop size distribution (DSD) and the relation between the physical model and radar parameters. The resulting radar rainfall relations based on this assumption have inherent parameterization errors. It is challenging to use these parametric rainfall relations to capture the complex space time variability in precipitation microphysics, especially in complex terrain. Even if the empirical relations are adaptively adjusted based on local DSD properties, such inherent errors are inevitable. Mismatch between radar rainfall estimates and pointwise gauge observations, the fourth category, is also critical in the assessment of radar QPE performance. This type of uncertainty ranges from fundamental radar and gauge mechanical functionality to the spatial and temporal differences in these two measuring techniques. Even if we assume gauges are a measure of truth and the point gauge data can represent the averaged radar estimates over a pixel at some height above the ground, a "residual error" is still expected due to the low level atmospheric turbulence including extreme wind effects.

In applications, it is difficult to quantify radar QPE errors from each category. Often, all types of errors in radar-based QPE product are evaluated and characterized as a whole. Numerous studies have focused on the correction of such overall bias in radar QPE using rain gauge data. For example, early work such as Brandes (1975) attempted to reduce the bias in radar estimates of rainfall using an adjustment factor. Krajewski (1987) developed an ordinary cokriging procedure to optimally merge radar rainfall estimates and rain gauge data. Test results based on numerical simulation of rainfall data at daily time scales showed the error characteristics of both radar and gauge as a function of gauge network density. Seo (1998) computed the expectation of rainfall at ungauged sites conditional on the observed rain gauge and radar data in order to account for the fractional coverage of rainfall, and subsequently introduced a mean field bias correction procedure (Seo et al. 1999) and a local bias correction procedure (Seo and Breidenbach 2002). A Bayesian technique was introduced by Todini (2001) to remove radar QPE error variance, which combines the use of block-kriging and Kalman filtering based on rain gauge measurements (representing the true rainfall field). Therein, the error structure of rain gauge and radar observations is estimated either from historical data with assumption of stationarity or real-time data with a parameter updating procedure. In the operational Multi-Radar Multi-Sensor (MRMS) system (Zhang et al. 2011), correction of hourly radar QPE is performed in three steps: 1) hourly rainfall differences between radar and gauge are calculated at gauge stations; 2) the differences are interpolated onto the MRMS grid via an inverse distance weighting scheme; 3) the interpolated difference field is subtracted from the hourly radar QPE estimates.

Roughly speaking, these bias correction methodologies can be classified into two categories, namely, mean field bias (MFB) correction and local bias (LB) correction. The MFB correction essentially is to estimate an overall bias in a mean sense for a large radar QPE domain, whereas the LB correction is to derive the radar QPE bias at each grid (i.e., resolution pixel). Depending on the specific application scenarios, both correction schemes have advantages and disadvantages. In general, one would expect that the MFB correction could better extract the overall radar QPE system bias, while the LB correction could better capture the spatial variations. Nevertheless, both approaches are purely based on statistical analysis (i.e., observed or estimated rainfall intensities). Environmental information such as terrain and temperature is not taken into account, although the bias corrected QPE was evaluated in different weather conditions. In complex terrain such as Northern California, wind direction and terrain forcing are among the key factors affecting the precipitation intensity and distribution (White et al. 2003). Conventional radar QPE bias correction schemes may not be able to capture the environmental variations as such.

This paper develops a hierarchical Bayesian framework to estimate the distribution of uncertainties associated with radar-derived rainfall products over complex 
terrain. In particular, the probability distribution of the targeted (i.e., true) precipitation conditional on the radar estimates is determined by Bayes' theorem. The radar product uncertainties are characterized and quantified in terms of various probability distributions conditional on the "true" measurements. The regional distribution of the model parameters are described using a generalized regression function that links radarbased estimates and ground references. In addition, the proposed Bayesian model is very flexible so that the terrain elevation and/or wind information can be easily incorporated in order to quantify the impact of orographic forcing on the variability of radar rainfall estimates. The Bayesian approach also takes into account the spatial and/or temporal covariates in order to get a full picture of the radar QPE uncertainty distributions. With such information, we can correct new radar estimates even without collocated gauge measurements in either temporal or spatial dimensions.

The remainder of this paper is organized as follows. Section 2 details the Bayesian model for radar QPE bias correction. Section 3 describes a simplified version of two conventional bias correction schemes for comparison. Case studies using operational radar hourly rainfall products at $1-\mathrm{km}$ scale are performed in section 4 to demonstrate the feasibility of the proposed Bayesian model. Section 5 quantifies the impact of terrain forcing on radar QPE correction within the Bayesian framework. Section 6 explores the potential of this new bias correction approach for operational applications, and the main findings of this study are summarized in section 7 .

\section{Bayesian framework for radar QPE bias correction}

\section{a. Generic model design}

Figure 1 shows an overall diagram of the proposed Bayesian framework for radar QPE bias correction. Therein, $T(s, t)$ denotes the "truth" of precipitation measurement at site $s$ and time $t$. For example, $T(s, t)$ can be assumed as the near-surface precipitation measurement at gauge station $s$ and the $t$ th hour in a day. The term $R(s, t)$ denotes the corresponding radar rainfall estimate at site $s$ and time $t$. Ideally, the revised radar rainfall estimate after correcting the bias in $R(s, t)$ should be close to $T(s, t)$. In this study, $t$ is on hourly scale. For simplicity, this paper uses $T$ and $R$ to represent $T(s, t)$ and $R(s, t)$, respectively.

According to Bayes' theorem, the conditional probability of $T$ given that radar estimate $R$ is available can be expressed as follows:

$$
P(T \mid R) \propto P(R \mid T) P(T),
$$

where $P(T \mid R)$ is the posterior probability of $T$ occurring given that $R$ is true; $P(R \mid T)$ is the likelihood of $R$ occurring given that $T$ is true; $P(T)$ is the prior probability of $T$.

Assuming that $T$ and $R$ are continuous since they both represent random precipitation values, the probability in Eq. (1) can be reformulated in terms of the conditional precipitation densities:

$$
f(T \mid R) \propto f(R \mid T) f(T),
$$

where $f(R \mid T)$ is the likelihood of radar estimate $R$ given ground reference $T$. It is also written as

$$
R \mid T \sim D(\boldsymbol{\theta}),
$$

where $D$ stands for the conditional distribution of $R \mid T$ derived from various distribution families, and $\boldsymbol{\theta}=$ $\left(\theta_{1}, \theta_{2}, \ldots, \theta_{n}\right)$ indicates the associated collection of $n$ distribution parameters (e.g., $n=2$ stands for normal or lognormal distribution; $n=3$ stands for generalized extreme value or Student's $t$ distribution).

Each conditional distribution $D$ can be expressed under the nonstationary assumption, in which the spatial and temporal covariates are considered: $\boldsymbol{\theta} \rightarrow g(\boldsymbol{\beta} ; T, \boldsymbol{\gamma})$, where $g$ is the linked function; $\boldsymbol{\beta}$ represents the parameter set of the linked function; $\boldsymbol{\gamma}$ is the collection of covariates (i.e., radar rainfall estimates, and/or terrain and wind information). Based on the calculated parameter set $\boldsymbol{\beta}$ from the training data, the quantity of interest can be computed at a new (testing) site and/or time. In particular, the radar QPE estimates at the new site and time are defined as $R^{*}$, and the obtained $q$ quantiles of the posterior predictive distribution of $T^{*}$ is used to correct $R^{*}$.

The posterior distribution of $T^{*}$ given $R^{*}$ occurring is

$$
f\left(T^{*} \mid R^{*}, T, R\right)=\int f\left(T^{*}, \boldsymbol{\beta} \mid R^{*}, T, R\right) d \boldsymbol{\beta} .
$$

Following the rule of joint probabilistic distributions, the right term inside the integral of Eq. (4) is written as

$$
f\left(T^{*}, \boldsymbol{\beta} \mid R^{*}, T, R\right)=f\left(T^{*} \mid R^{*}, T, R, \boldsymbol{\beta}\right) f\left(\boldsymbol{\beta} \mid R^{*}, T, R\right) .
$$

Because $T^{*}$ is independent from $T$ and $R$, the first term of the right hand side of Eq. (5) is transformed as

$$
f\left(T^{*} \mid R^{*}, T, R, \boldsymbol{\beta}\right)=f\left(T^{*} \mid R^{*}, \boldsymbol{\beta}\right) .
$$

Since the parameters $\boldsymbol{\beta}$ are independent from the new estimate $R^{*}$, the second term on the right hand side of Eq. (5) can be expressed as

$$
f\left(\boldsymbol{\beta} \mid R^{*}, T, R\right)=f(\boldsymbol{\beta} \mid T, R) .
$$




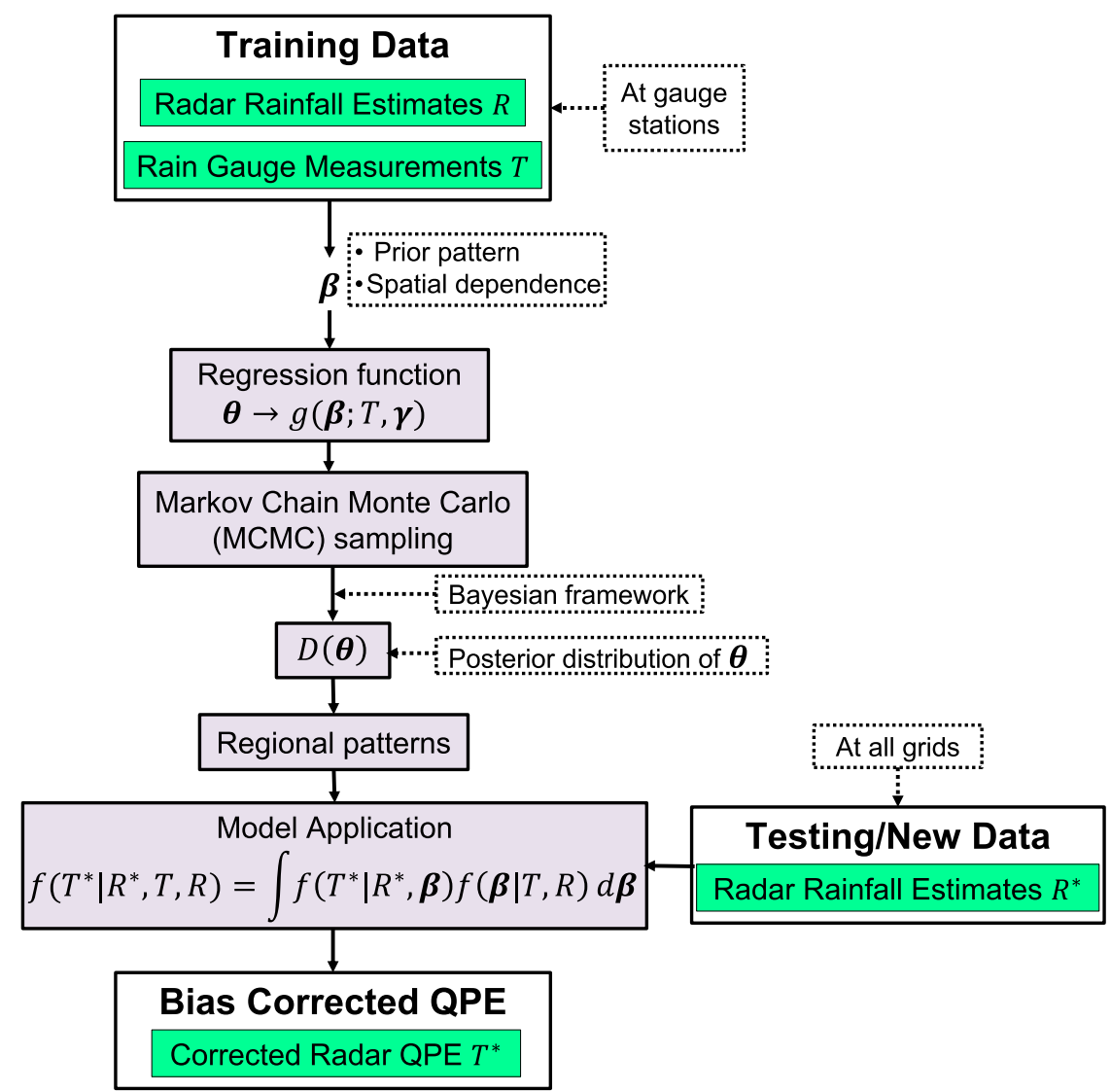

FIG. 1. Overall diagram of the proposed Bayesian framework for radar QPE bias correction. Symbols are defined in the text. Note that, although the uncertainty parameters are estimated based on gauge data at point locations, application of the trained framework is not limited to those specific locations.

Hence, the posterior predictive distribution of $T^{*}$ in Eq. (4) can be written as

$$
f\left(T^{*} \mid R^{*}, T, R\right)=\int f\left(T^{*} \mid R^{*}, \boldsymbol{\beta}\right) f(\boldsymbol{\beta} \mid T, R) d \boldsymbol{\beta},
$$

which forms the fundamental of the Bayesian-based radar QPE bias correction scheme.

\section{b. Implementation of the Bayesian framework}

The empirical distribution of $R$ conditional on $T$ [i.e., Eq. (3)] should be fitted empirically from the relationship between radar rainfall estimates, ground reference and relevant spatiotemporal covariates in specific applications. In general, it can be assumed to be a lognormal distribution (e.g., Cho et al. 2004) or Student's $t$ distribution. For demonstration purposes, this study adopts the Student's $t$ distribution, with its second parameter expressed as a linear regression of the estimate $R$. Here, it should be noted that we have also tried other distributions including lognormal. No significant differences were observed in the performance of final (bias corrected) radar QPE products for the case studies presented in this analysis. Extensive comparison of different distribution assumptions is beyond the scope of this paper.

Two regression models are considered. The first one is only based on the intensity values of radar rainfall estimates (i.e., no environmental factors). The second one uses both radar rainfall estimates and terrain topography. In the first scenario, the empirical distribution of $R$ conditional on $T$ is expressed as

$$
\begin{aligned}
R \mid T & \sim \operatorname{Student}\left(\nu, \alpha_{1}+\alpha_{2} R, \sigma\right), \\
\nu, \alpha_{1}, \alpha_{2} & \sim \operatorname{Uniform}(-\infty,+\infty), \\
\sigma & \sim \operatorname{Uniform}(0,+\infty),
\end{aligned}
$$

where $\nu$ is the number of degrees of freedom; $\left(\alpha_{1}+\alpha_{2} R\right)$ is a linear relation of the radar estimate $R$, representing the sample mean; and $\sigma$ is the variance. The distributions of $\nu, \alpha_{1}, \alpha_{2}$, and $\sigma$ in Eqs. (9b) and (9c) essentially means that they are real numbers. 
The empirical distribution of $R$ conditional on $T$ for the second scenario is similar to Eq. (9). But the terrain elevation information is considered in the linear relation (i.e., sample mean):

$$
\begin{aligned}
R \mid T & \sim \operatorname{Student}\left[\nu,\left(\alpha_{1}+\beta_{1}\right)+\left(\alpha_{2}+\beta_{2}\right) R, \sigma\right], \\
\nu, \alpha_{1}, \alpha_{2} & \sim \operatorname{Uniform}(-\infty,+\infty), \\
\sigma & \sim \operatorname{Uniform}(0,+\infty),
\end{aligned}
$$

where $\beta_{1}$ and $\beta_{2}$ denote the impact of terrain forcing, which follows the multivariate normal distribution as follows:

$$
\left(\begin{array}{l}
\beta_{1} \\
\beta_{2}
\end{array}\right) \sim N\left(\begin{array}{l}
0 \\
0
\end{array}, \Sigma_{\beta}\right)
$$

In the radar QPE bias correction process, the distribution is bivariate because of the two covariances between the radar estimate and terrain.

It should be noted again that the designed Bayesian model is very flexible. One can easily incorporate additional environmental factors. For example, to study the impact of wind on radar QPE performance, the model can be extended to include a term for wind information as follows:

$$
\begin{aligned}
R \mid T \sim \operatorname{Student}\left[\nu,\left(\alpha_{1}+\boldsymbol{\beta}_{1}+\boldsymbol{\gamma}_{1}\right)\right. \\
\left.+\left(\alpha_{2}+\boldsymbol{\beta}_{2}+\boldsymbol{\gamma}_{2}\right) R, \sigma\right], \\
\nu, \alpha_{1}, \alpha_{2} \sim \operatorname{Uniform}(-\infty,+\infty), \\
\sigma \sim \operatorname{Uniform}(0,+\infty),
\end{aligned}
$$

where $\boldsymbol{\gamma}_{1}$ and $\boldsymbol{\gamma}_{2}$ denote the impact of wind. As a result, both $\left(\boldsymbol{\beta}_{1} / \boldsymbol{\beta}_{2}\right)$ and $\left(\boldsymbol{\gamma}_{1} / \boldsymbol{\gamma}_{2}\right)$ will follow multivariate normal distributions:

$$
\begin{aligned}
& \left(\begin{array}{l}
\boldsymbol{\beta}_{1} \\
\boldsymbol{\beta}_{2}
\end{array}\right) \sim \boldsymbol{N}\left(\begin{array}{c}
0 \\
0
\end{array}, \boldsymbol{\Sigma}_{\beta}\right), \\
& \left(\begin{array}{l}
\boldsymbol{\gamma}_{1} \\
\boldsymbol{\gamma}_{2}
\end{array}\right) \sim \boldsymbol{N}\left(\begin{array}{l}
0 \\
0
\end{array}, \boldsymbol{\Sigma}_{\gamma}\right),
\end{aligned}
$$

where $\boldsymbol{\Sigma}_{\beta}$ and $\boldsymbol{\Sigma}_{\gamma}$ are the covariance matrices.

Nevertheless, wind information is only available at a small portion of gauge stations used in this study. It is challenging to fully understand the wind impact on radar QPE with limited wind observations. As such, this paper only focuses on demonstration of the two models illustrated in Eqs. (9) and (10).

\section{c. Parameter estimation}

The prediction of the posterior $T^{*}$ (i.e., bias corrected radar QPE) in Eq. (8) is nontrivial since the empirical distribution $f$ is rather arbitrary. In addition, it is difficult to obtain an analytic form of the posterior predictive distribution since there is no general way to calculate the associated integral in Eq. (8). To this end, this study uses the Markov chain Monte Carlo (MCMC) technique to produce the posterior distribution of each parameter and quantify the associated predictive uncertainty (Carpenter et al. 2017). The model parameters and the spatial-temporal covariates of terrain component in radar QPE are routinely updated via the MCMC experiments. In particular, it is assumed that there are many replicates of the MCMC samples for $\boldsymbol{\beta}$ in Eq. (8), and the prediction of the posterior target $T^{*}$ in Eq. (8) is performed for each sample. The collection of all samples is a realization of posterior predictive distribution for $T^{*}$ at a test site/grid, where the input data include estimated parameters $\boldsymbol{\beta}$ and the known radar estimate $R^{*}$.

To sample $T^{*}$ from $f\left(T^{*} \mid R^{*}, T, R\right)$ in Eq. (8), the MCMC technique is used again, but $\boldsymbol{\beta}, R^{*}$ are constant this time. Ultimately, we can obtain the spatial pattern of bias corrected radar QPE in terms of the mean, median, and credible intervals in the regions of interest.

\section{Conventional radar QPE bias correction approaches for comparison}

As mentioned previously, conventional statistical approaches to radar QPE bias correction mainly include MFB and LB correction. In the following section, both are briefly reviewed and will be compared with the proposed Bayesian framework.

\section{a. MFB correction}

The MFB correction is performed through the estimation of an adjustment factor that is defined as the ratio of gauge observed rainfall and the rainfall estimated from radar $(G / R)$. This bias-adjustment factor is estimated as

$$
\mathrm{MFB}=\frac{\sum_{i=1}^{N} G_{i}}{\sum_{i=1}^{N} R_{i}}
$$

where $G_{i}$ and $R_{i}$ represent gauge and radar rainfall measurements, respectively; $N$ stands for the number of valid radar-gauge pairs. More detailed estimation of such bias ratio can be found in Seo et al. (1999) and Chumchean et al. (2006). Then the radar rainfall estimates are corrected by multiplying the bias-adjustment factor MFB. This is the simplest way to reduce the average bias between radar estimates and corresponding gauge rainfall amounts at the rain gauge locations. 
In this paper, the number $N$ is determined at an event scale, and the calculated MFB is implemented on an hourly basis. That is, both $G_{i}$ and $R_{i}$ in Eq. (14) will represent hourly rainfall estimates, and the valid radargauge pairs during a whole event are considered when calculating the bias ratio.

\section{b. LB correction}

The local bias correction scheme implemented in this study is similar to that adopted by the operational MRMS system (Zhang et al. 2011). First, an additive error in the radar rainfall estimate is calculated at each gauge location:

$$
e_{j}=R_{j}-G_{j},
$$

where $R_{j}$ and $G_{j}$ are radar rainfall estimate and gauge rainfall observation at the $j$ th gauge location, respectively; $e_{j}$ stands for the rainfall difference between radar and gauge. Then, the differences at multiple gauge stations are interpolated over the predefined radar QPE domain according to the following criterion:

$$
\begin{aligned}
& R_{b}=\frac{\sum_{j=1}^{n} e_{j} w_{j}}{\sum_{j=1}^{n} w_{j}}, \\
& w_{j}=\left\{\begin{array}{cc}
1 / d_{j}^{x}, & d_{j} \leq D \\
0, & d_{j}>D
\end{array}\right.
\end{aligned}
$$

where $R_{b}$ is the estimated radar QPE bias at a certain grid point; $w_{j}$ is the weight assigned to the $j$ th gauge; $d_{j}$ is the distance between the grid point and the $j$ th gauge. Rain gauges located far from the grid point will have small weights and thus little effect on the bias estimate. The term $x$ is an exponent, which varies from 0.5 to 3 and is determined each hour through cross validation that minimizes the interpolation error of radar-gauge differences in a particular domain; $n$ is the total number of gauge-radar pairs within a specified radius of influence (i.e., $D$ ). Note that the index $j$ in Eqs. (15) and (16) denotes the gauge identities in the spatial domain, whereas the index $i$ in Eq. (14) can represent both spatial and temporal dimensions.

In this study, the MRMS approach is adopted to select initial values of $x$ and $D$. This choice is also motivated by the ultimate goal of extending the proposed Bayesian framework to the operational MRMS system. In particular, the cross validation is performed by removing a rain gauge and interpolating to its location using radargauge errors at all the remaining rain gauges. The difference between the interpolated radar-gauge error and the observed value is then calculated. After cross validating all rain gauge points, a total cross-validated mean-squared error is determined. The two parameters are then adjusted to a new set of trial values and the cross-validation process is repeated. Cross validation is performed for each analysis time (i.e., every hour), resulting in different optimum parameters each hour. This gauge-based local bias correction provided consistent improvements over the radar-only QPE across the CONUS domain (Zhang et al. 2011).

\section{Case studies in Northern California}

\section{a. Study domain and precipitation event}

For initial verification of the proposed Bayesian correction approach, this paper uses the Russian River watershed in Northern California as a demonstration study domain. Figure 2 illustrates the detailed terrain information in the vicinity of this watershed, as well as rain gauge stations deployed as part of the National Oceanic and Atmospheric Administration (NOAA) Hydrometeorology Testbed (HMT) (White et al. 2013). It is one of the most flood-prone areas in the State of California because of the watershed's unique geography. Accurate QPE is critical to balance the competing needs for water supply and flood mitigation in this particular domain. However, it is very difficult to obtain reliable QPE using operational Weather Surveillance Radar-1988 Doppler (WSR-88D) due to the sampling limitations of WSR-88D and complex precipitation microphysics resulting from orographic enhancement (Willie et al. 2017; Cifelli et al. 2018; Bytheway et al. 2019).

The radar-only rainfall products produced by the operational MRMS system are used as a basis for applying various bias correction methodologies. The radar-only product essentially is hourly rainfall accumulations created on $0.01^{\circ} \times 0.01^{\circ}$ latitude/longitude grids. Interested readers are referred to Zhang et al. (2011) for more details about the algorithms implemented by the MRMS system. The MRMS radaronly product is selected in this study mainly for two reasons: 1) the MRMS is an operational system and MRMS products are widely used in the precipitation community; 2) it is commonly recognized that the performance of MRMS products over the western United States (i.e., west of the Rockies) is poor, and the bias correction scheme currently adopted in the MRMS system (see also section 3b) may not be sufficient to represent the environmental variations in complex terrain. In addition, the findings in this study are expected to support future development of the MRMS system. 


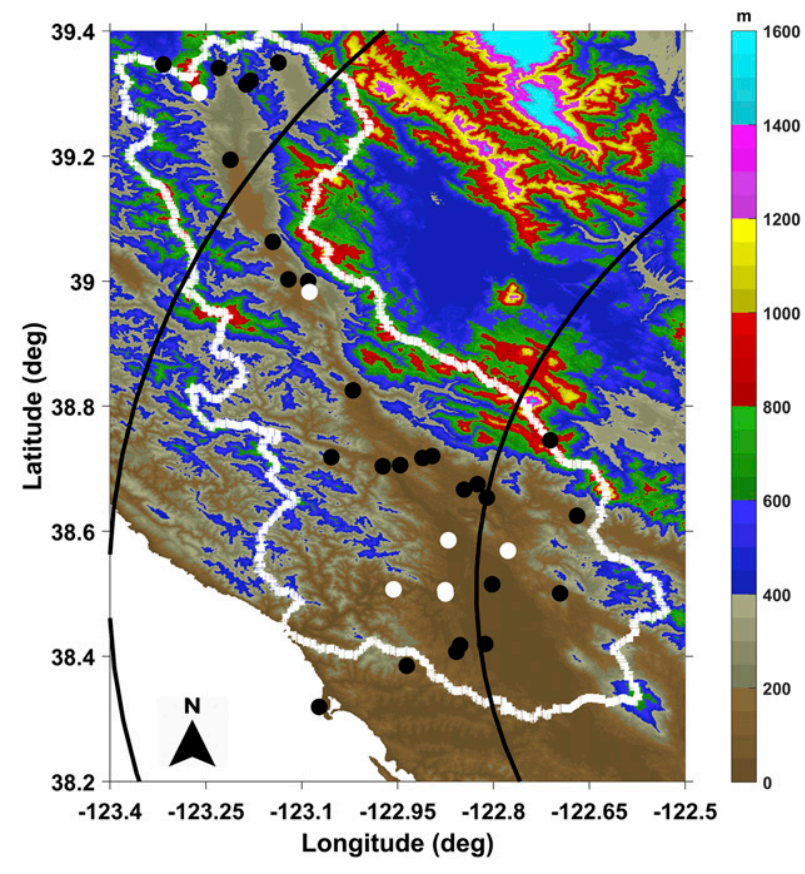

FIG. 2. The digital elevation model (DEM) information near the Russian River basin in Northern California. The basin boundaries are highlighted in white. The black (training sites) and white (validation sites) dots denote NOAA rain gauge locations. The split of training and validation sites is random, and this figure shows one combination for illustration purposes. The nearest NEXRAD radar [Sacramento, CA $(\mathrm{KDAX})]$ is located at $38.5011^{\circ} \mathrm{N}$, $121.6778^{\circ} \mathrm{W}$; range rings of 100 and $200 \mathrm{~km}$ are indicated by black curves.

As illustrated in Fig. 2, there are 34 NOAA gauges deployed near the Russian River basin, which are assumed as ground truths when correcting the radarderived rainfall products. More details about the locations of these gauge sites are available in Chen et al. (2019b). Each site is equipped with a 10-m surface meteorological tower and a tipping-bucket rain gauge that measures rainfall every two minutes with 0.01 -in. (i.e., $0.254 \mathrm{~mm}$ ) resolution. NOAA engineers at the Earth System Research Laboratory (ESRL) regularly maintain the surface meteorological sites to ensure the data quality. At each station, the 2-min rainfall data are postprocessed at NOAA/ESRL using an automated quality-control routine designed to remove obvious outliers. In this demonstration study, the 2-min gauge data are aggregated to hourly accumulations at the top of each hour to match the MRMS product resolution. The gauge stations are randomly split into calibration ( $80 \%)$ and validation $(20 \%)$ sites. The calibration sites are used to derive the parameters in the specific models described in section $2 \mathrm{~b}$ [or more generally the parameter $\boldsymbol{\beta}$ in Eq. (8)] and associated uncertainties in the radar rainfall estimates. The radar estimates are then corrected at all grid points based on the derived uncertainty parameters. The bias corrected radar estimates at the validation gauge sites are evaluated using corresponding validation gauge data.

In the following section, results for a precipitation event that occurred from 7 to 10 February 2017 are detailed to prototype the Bayesian method and benchmark the performance relative to the MFB and LB correction techniques. Northern California's Mediterranean climate is characterized by wet winters and dry summers, and it relies on a few atmospheric river (AR) events every year for water supply (Ralph et al. 2006; Dettinger 2011). This event was a typical AR storm which can represent the local precipitation characteristics to a large extent. This event also coincided with the Oroville Dam Crisis (White et al. 2019), resulted in major flooding across portions of Northern California.

\section{b. Results}

In this section, the Bayesian model in Eq. (9) is evaluated first, which is only based on the rainfall intensities (i.e., no environmental information included such as terrain). The goal is to determine if the Bayesian technique can outperform the conventional radar QPE bias correction approaches described in section 3 .

As mentioned, the split of gauge stations into calibration versus validation sites is determined randomly in order to fully capture the distribution of QPE uncertainties. Although the gauge proportion of $80 \%$ for calibration and $20 \%$ for validation are not changed, the specific site selections are different for each experiment. Even if the validation and calibration gauges are determined in one experiment, the parameter $\boldsymbol{\beta}$ in Eq. (8) has many realizations (100 in our study) based on the MCMC samples. For each realization, the Bayesian framework will produce a bias corrected estimate. For illustration purposes, this paper only presents results for one experiment. That is, the 27 stations denoted by black dots in Fig. 2 are used as calibration sites, whereas the seven stations denoted by white dots are used for validation purpose. It should be noted that in total 10 experiments (not shown) have been conducted with different combinations of calibration and validation gauges. We did observe some differences in the bias correction performance after changing the combination of gauge sites, especially when the calibration sites are accidentally assembled in a small area. But overall, they all show similar positive performance. In addition, for each experiment, the median value of the 100 versions of the bias corrected radar QPE at each grid point (not limited to gauge locations) is selected and considered the final correction result at each location. 

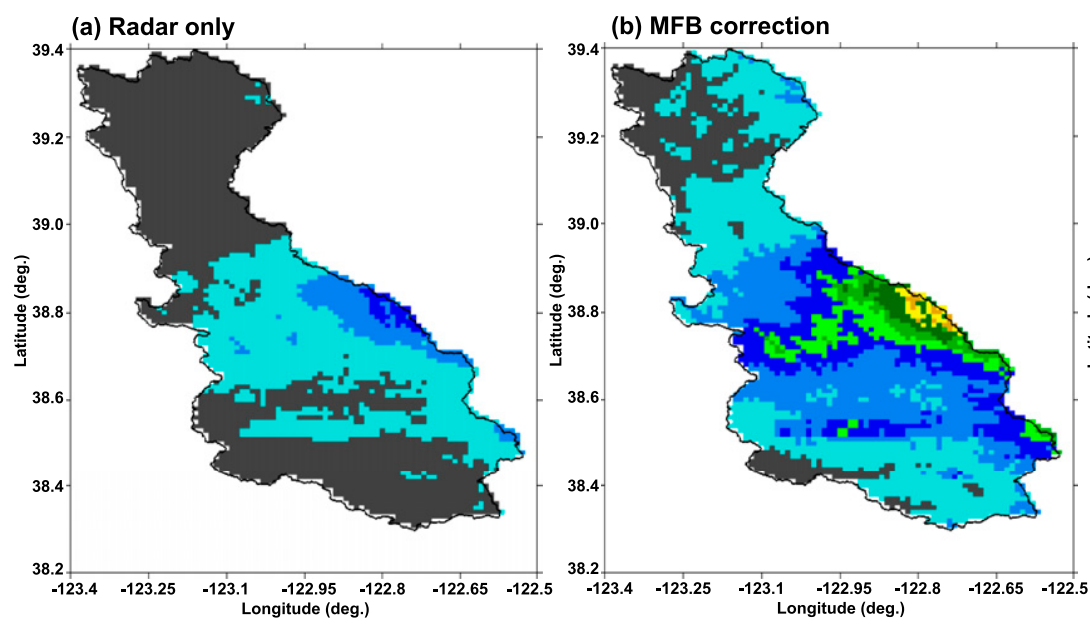

(c) LB correction

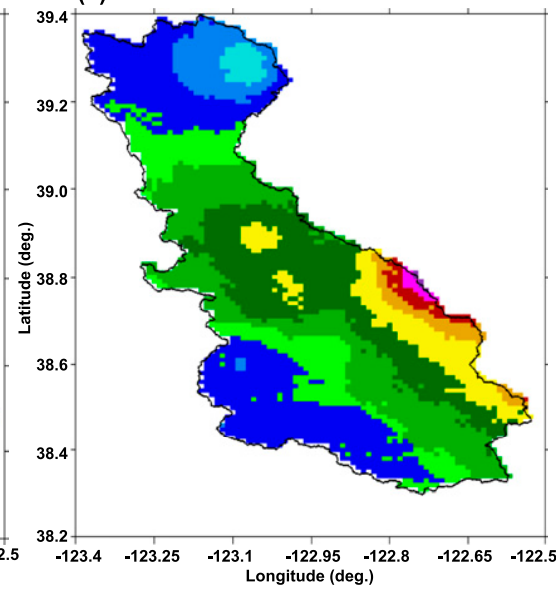

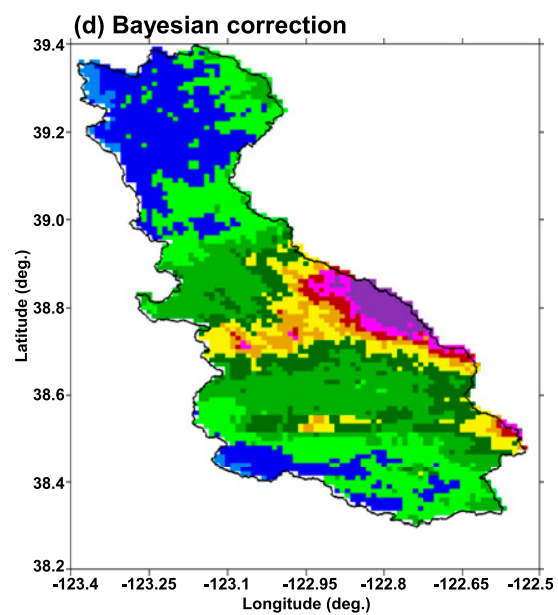

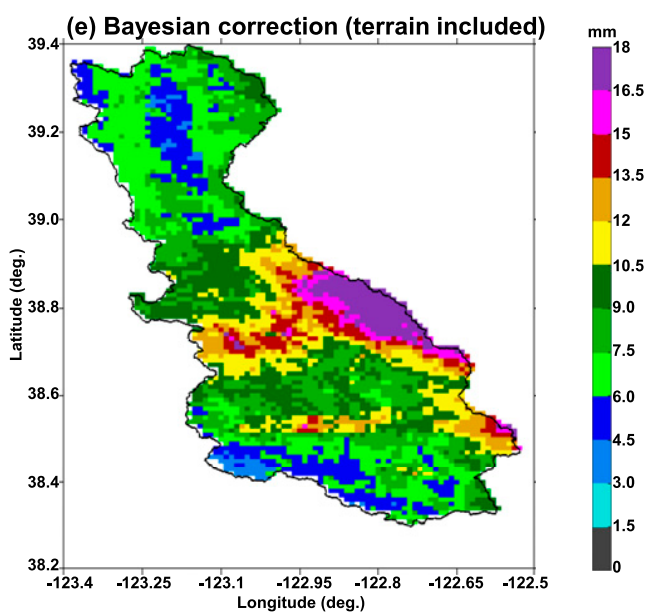

FIG. 3. Radar-derived hourly rainfall estimates over the Russian River watershed before and after bias corrections at 1300 UTC 7 Feb 2017: (a) radar-only estimates; (b) estimates after MFB correction; (c) estimates after LB correction; (d) estimates after Bayesian-based bias correction; and (e) as in (d), but with terrain information included.

Figure 3 illustrates an example of radar-derived hourly rainfall estimates over the Russian River watershed before and after bias corrections at 1300 UTC 7 February 2017. Apparently, the radar estimated rainfall intensity is substantially enhanced after applying various bias correction techniques, especially the Bayesian-based approach. Figure 4 shows the hourly rainfall estimates and rainfall accumulations at a selected validation gauge location during 13 intense rain hours for the February 2017 precipitation event. The red line denotes rain gauge observations. The black and blue lines respectively represent radar-only estimates before and after bias correction using the proposed Bayesian technique. The bias corrected results using conventional MFB (magenta line) and LB (green line) approaches are also shown in Fig. 4. As expected, the radar-only product shows significant underestimation compared to gauge observations, especially after 0600 UTC. After bias correction using the MFB and LB correction approaches, both show overestimation in terms of accumulated rainfall. By contrast, the bias corrected results based on the Bayesian technique agree very well with rain gauge observations.

Figure 5 shows the scatterplots of hourly rainfall estimates from radar versus gauge measurements at all seven validation gauge stations. Linear fittings of various estimates and their $95 \%$ confidence intervals are also indicated in Fig. 5. Overall, the radar-only estimates perform poorly, demonstrating the challenges of radar QPE over complex terrain. After the MFB correction, the severe underestimation is alleviated, but the corrected estimates are still far from the collocated gauge observations. Both the LB correction and Bayesian technique substantially improve the radar QPE performance. In particular, the corrected results based on the proposed Bayesian method agree better with gauge 


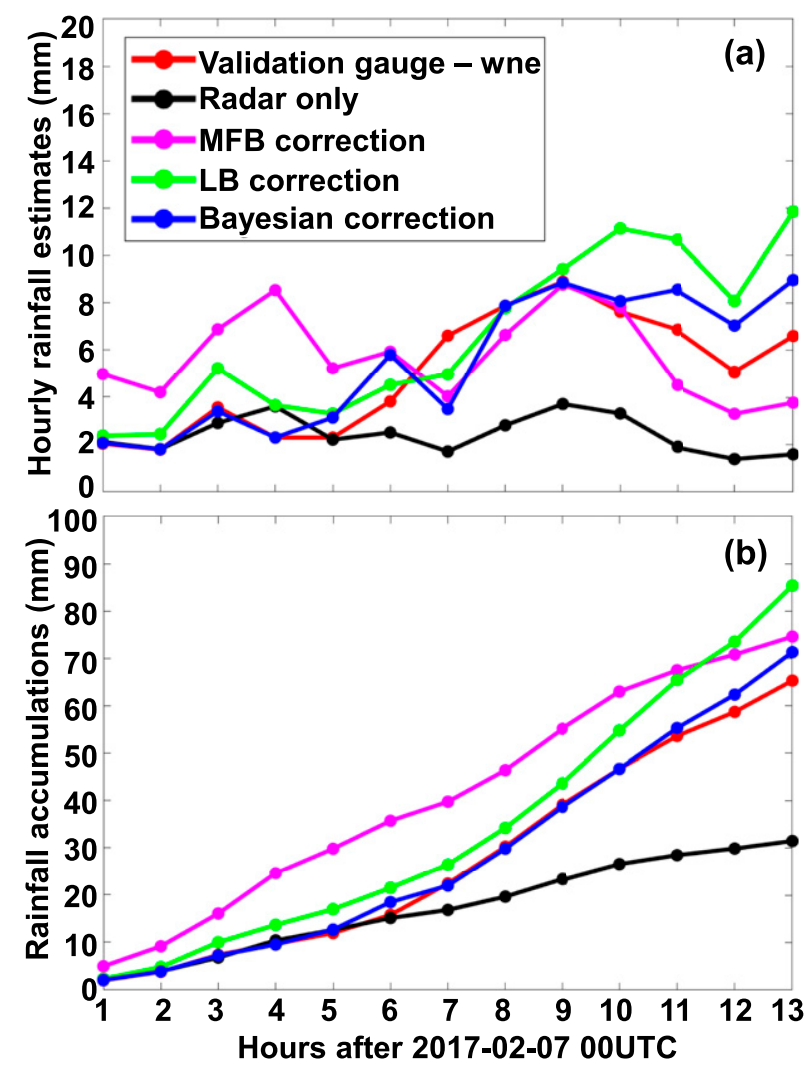

FIG. 4. Hourly rainfall estimates and rainfall accumulations at a selected validation gauge location during the February 2017 precipitation event: (a) hourly rainfall estimates and (b) rainfall accumulations. Estimates at other validation gauge locations are not shown since they show similar performance.

measurements, and the $95 \%$ confidence interval is relatively narrower, indicating smaller variation in the correction performance.

The probability density functions (PDFs) of gauge measurements, radar-only estimates, and the bias corrected radar estimates using the three different techniques are illustrated in Fig. 6. Again, compared to gauge observations, the radar-only estimates show very poor performance, with the distribution highly skewed to low rainfall intensities relative to the gauge distribution. For rainfall intensities less than $3 \mathrm{~mm} \mathrm{~h}^{-1}$, all three correction methods show compatible performance. However, the Bayesian approach shows better performance at higher intensities, although the LB correction results seem able to capture the heavy rainfall values $\left(>12 \mathrm{~mm} \mathrm{~h}^{-1}\right)$ slightly better in this particular event.

To further quantify the bias correction performance, the following metrics are computed for the combined observations at the seven validation gauge locations, including the root-mean-square error (RMSE; mm), normalized mean error (NME; \%), normalized mean

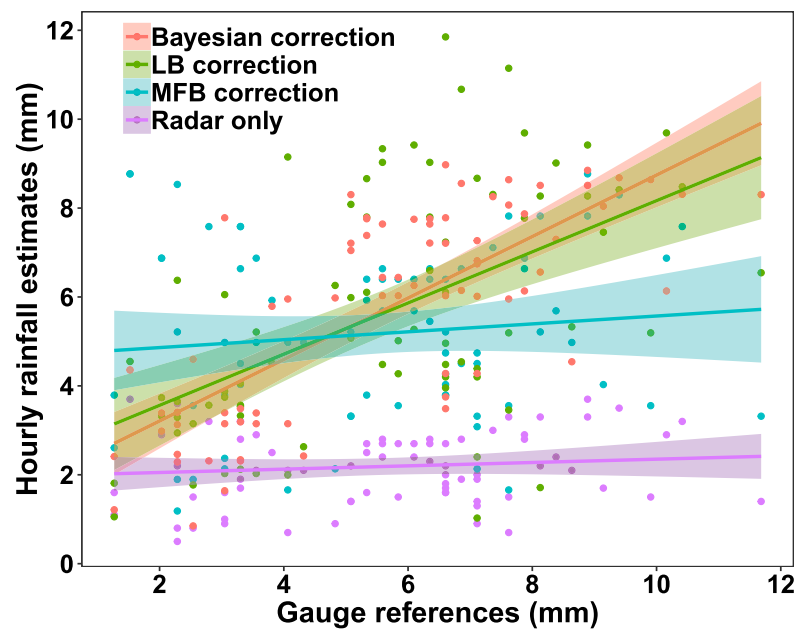

FIG. 5. Scatterplot of hourly rainfall estimates from radar vs validation gauge observations during the February 2017 precipitation event. The validation gauge locations are indicated by white dots in Fig. 2. The radar estimates include radar only and bias corrected products using the various techniques described in the text. The black solid line represents the 1:1 line, whereas other lines indicate the linear fitting of various estimates, and the shading areas represent corresponding $95 \%$ confidence intervals.

absolute error (NMAE; \%), and the Pearson's correlation coefficient (CORR):

$$
\begin{aligned}
\text { RMSE } & =\sqrt{\left\langle\left(R_{R}-R_{G}\right)^{2}\right\rangle}, \\
\mathrm{NME} & =\frac{\left\langle R_{R}-R_{G}\right\rangle}{\left\langle R_{G}\right\rangle} \times 100 \%,
\end{aligned}
$$

$\mathrm{NMAE}=\frac{\left\langle\left|R_{R}-R_{G}\right|\right\rangle}{\left\langle R_{G}\right\rangle} \times 100 \%$,

$\operatorname{CORR}=\frac{\sum\left[\left(R_{R}-\left\langle R_{R}\right\rangle\right)\left(R_{G}-\left\langle R_{G}\right\rangle\right)\right]}{\sqrt{\sum\left(R_{R}-\left\langle R_{R}\right\rangle\right)^{2}} \sqrt{\sum\left(R_{G}-\left\langle R_{G}\right\rangle\right)^{2}}}$,

where $R_{R}$ and $R_{G}$ are the rainfall estimate from radar and validation gauges, respectively; the angle brackets stand for the sample average.

The overall evaluation results at the seven validation gauge stations are illustrated in Fig. 7. In particular, the RMSEs (lower values indicate better performance) of radar-only, MFB corrected, LB corrected, and Bayesianbased correction products are $4.20,2.99,2.48$, and $1.71 \mathrm{~mm}$, respectively. The NMEs are $-60.7 \%,-6.8 \%, 1.0 \%$, and $2.0 \%$ for radar-only, MFB corrected, LB corrected, and Bayesian-based correction products, respectively. Here, it should be noted that the NME values only indicate the overall biases. Possible cancellation of positive and negative biases may exist, resulting in a misleading performance. Combined with the CORR, which describes the 


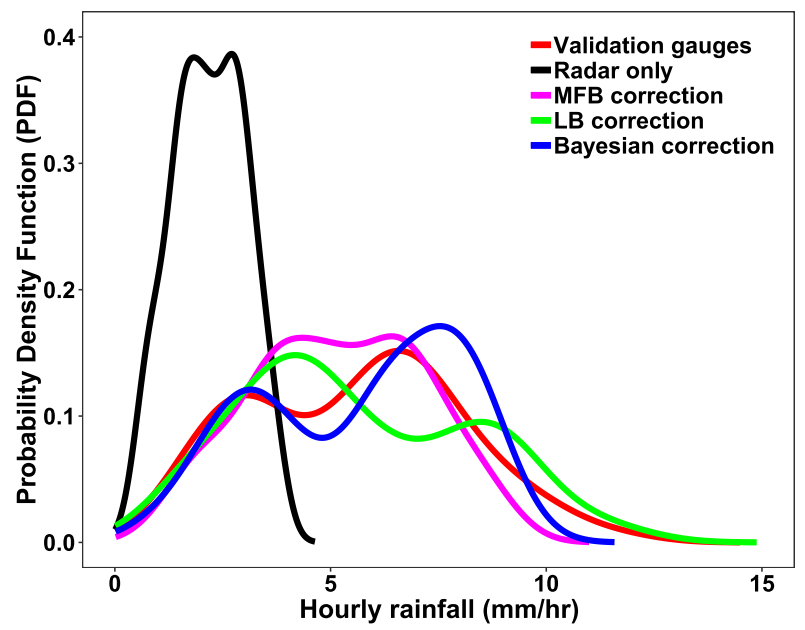

FIG. 6. Probability density functions (PDFs) of hourly rainfall estimates (shown in Fig. 5) from radar and validation gauges.

agreement between radar estimates and gauge observations, the NME is more useful. The CORRs (higher values indicate better performance) are $0.11,0.11,0.53$, and 0.74 for the four estimates, respectively. In addition, the NMAEs (the lower the better) can better reflect the performance of various products, which are $64.5 \%, 41.0 \%, 35.0 \%$, and $24.2 \%$ for the four estimates, respectively.

\section{Impact of terrain forcing on radar QPE performance}

Previous studies such as White et al. (2003) concluded that wind direction and terrain forcing were among the primary mechanisms responsible for precipitation in such complex terrain. As such, this paper also attempts to resolve the impact of terrain topography on the radar QPE bias correction performance. As detailed in section $2 \mathrm{~b}$, the designed Bayesian framework is very flexible in terms of including additional environmental information. In this section, the regression model in Eq. (10) is used in the Bayesian correction analysis. In this model, the terrain elevation is considered a factor contributing to the radar rainfall estimates. Ideally, wind information could be included as well. However, we decided not to do so due to the very limited wind observations available in this study. In future, wind information derived from models like the High-Resolution Rapid Refresh (HRRR) (Benjamin et al. 2016) may be useful in extending the Bayesian framework to further quantify different contributing factors in the radar-derived precipitation products.

Given the specific locations of NOAA HMT gauges (see also Chen et al. 2019b), the terrain information is classified into four categories based on the terrain elevation. In particular, all the radar QPE grid points lower than $50 \mathrm{~m}$ in elevation (MSL) are labeled as one category; those between 50 and $100 \mathrm{~m}$ are labeled as the second category; those between 100 and $300 \mathrm{~m}$ are labeled as the third category; and those higher than $300 \mathrm{~m}$ are labeled as the fourth category. Such labeling is incorporated as $\beta_{1}$ and $\beta_{2}$ in the regression model of Eq. (10). For illustration purposes, the same experiment as section 4 is conducted (i.e., the same groups of 27 calibration and seven validation sites are selected) to emphasize the impact of terrain information.

Scrutinizing the DEM information in Fig. 2 and the example radar QPEs in Figs. 3d and 3e, we can see that the rainfall estimates at high elevations are further enhanced after incorporating the terrain information in the Bayesian-based bias correction framework. Similar to (a) RMSE (mm)

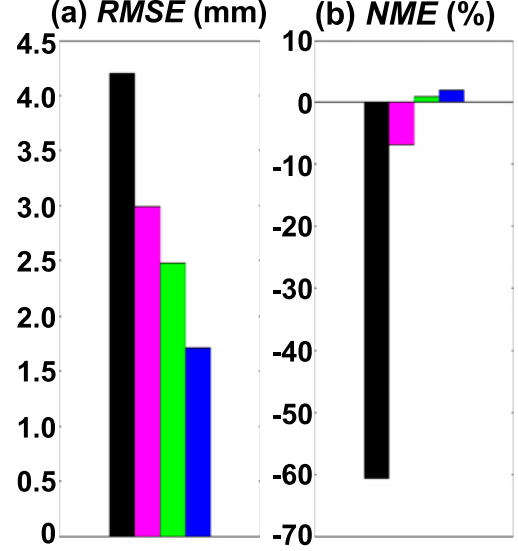

(c) NMAE (\%)

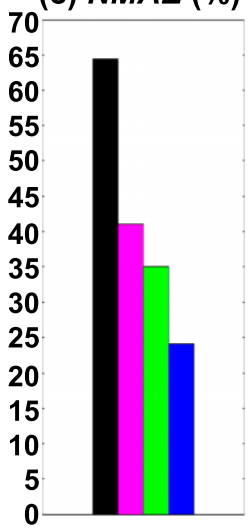

(d) CORR

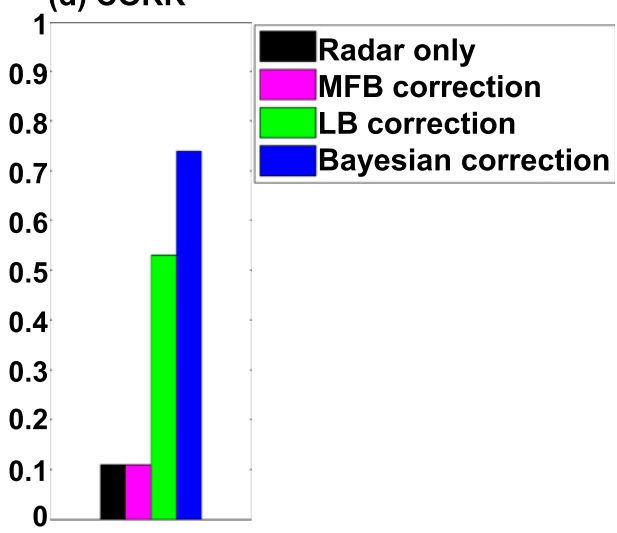

FIG. 7. Quantitative evaluation results of hourly rainfall estimates from radar before and after bias correction using different techniques for the 7 Feb 2017 precipitation event: (a) RMSE (mm), (b) NME (\%), (c) NMAE (\%), and (d) CORR. 


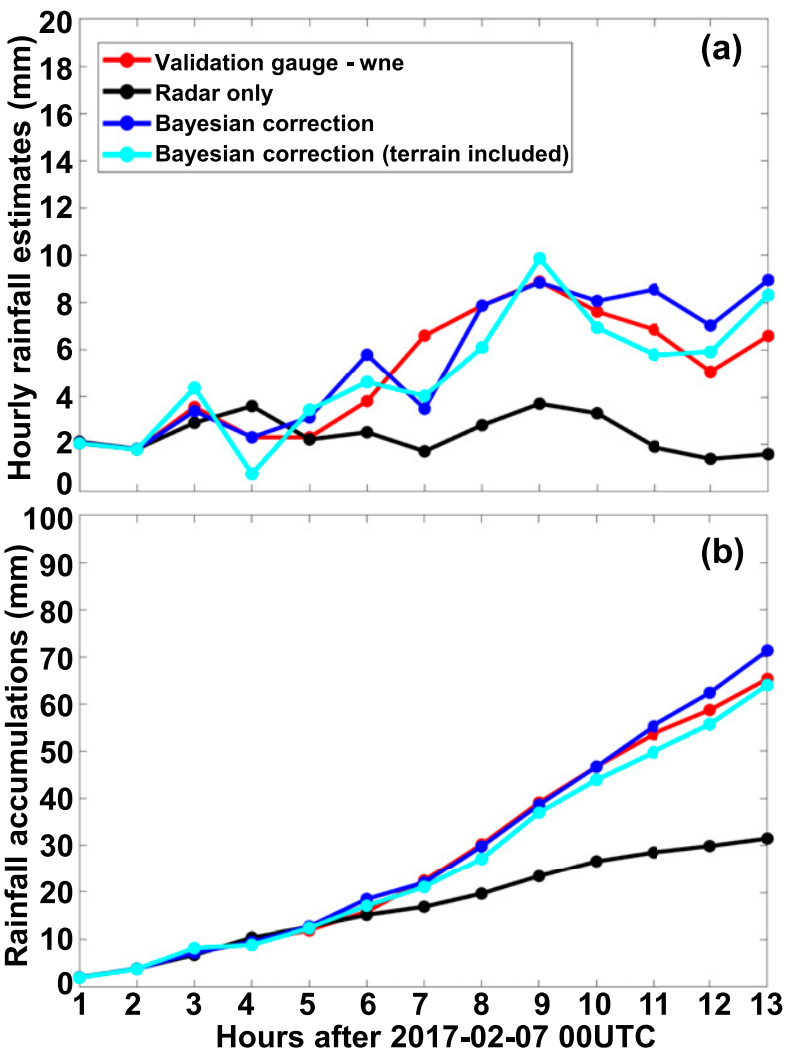

FIG. 8. As in Fig. 4, but including the impact of terrain information in the Bayesian correction technique: (a) hourly rainfall estimates and (b) rainfall accumulations.

Fig. 4 which shows the superior performance of the Bayesian technique to conventional MFB and LB correction approaches, Fig. 8 illustrates the bias corrected results at the same validation gauge station. The MFB and LB corrected products are not included in Fig. 8 since they are already shown in Fig. 4. Rather, only the correction results based on the Bayesian technique with and without including the terrain information are presented. At this specific location, noticeable differences are observed in the corrected hourly rainfall estimates (Fig. 8a) before (blue line) and after (cyan line) considering the terrain information. However, the differences in the accumulated rainfall (Fig. 8b) are small with the Bayesian method including terrain information performing slightly better.

Figure 9 shows the scatterplots of hourly rainfall estimates at all seven validation gauge stations. The linear fittings of the corrected rainfall estimates and their $95 \%$ confidence intervals are included as well. Figure 9 shows that, after taking into account the terrain topography, the linear fitting curve is closer to the 1:1 line, demonstrating the improved performance of the Bayesian method with terrain information included. The PDFs of

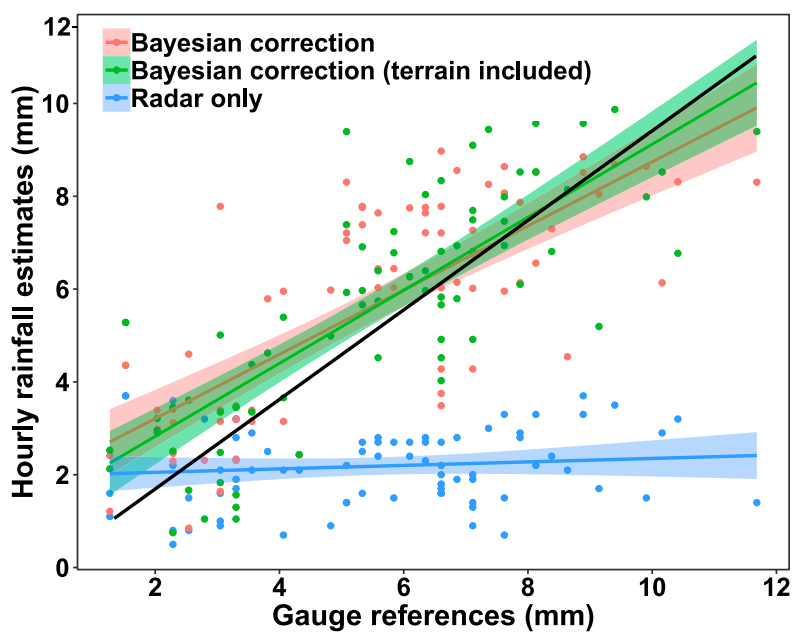

FIG. 9. Scatterplots of radar-only rainfall estimates and bias corrected estimates using the proposed Bayesian approach with and without including the terrain information, vs validation gauge observations. The black solid line represents the 1:1 line, whereas other lines indicate the linear fitting of various estimates, and the shading areas stand for corresponding $95 \%$ confidence intervals.

hourly rainfall estimates shown in Fig. 9 are illustrated in Fig. 10. Surprisingly, the bias corrected result after incorporating the terrain information misses the first, low intensity peak $\left(\sim 3 \mathrm{~mm} \mathrm{~h}^{-1}\right)$ of the rainfall distribution. This may reflect the relative impact of terrain information on the distribution of rainfall intensity but more research is needed to confirm this hypothesis. Overall, the Bayesian method including the terrain information shows an improved distribution compared to validation gauge observations, especially at moderate to heavy rain regions.

Table 1 shows the quantitative evaluation results of corrected hourly rainfall estimates before and after incorporating the terrain information. At the seven validation gauge sites, all the evaluation scores are improved after including the terrain factor: RMSE improves from 1.71 to $1.59 \mathrm{~mm}$; NME improves from $2.0 \%$ to $1.2 \%$; NMAE improves from $24.2 \%$ to $22.5 \%$; and CORR improves from 0.74 to 0.79 . Compared to the radar-only products, the QPE performance is dramatically improved after applying this Bayesian-based bias correction.

\section{Implications for real-time operational applications}

It is worth noting that the separation of calibration and validation gauges in this paper is only for the purpose of independent verification. In future operational implementations, there is no need to divide the gauge data into different groups. It is recommended that all the 


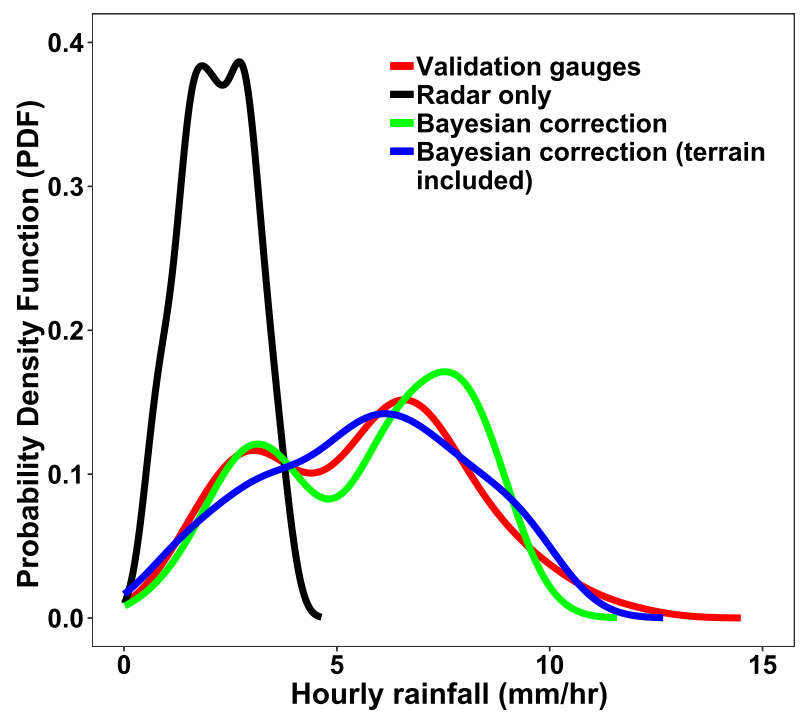

FIG. 10. PDFs of hourly rainfall estimates shown in Fig. 9.

quality-controlled gauges be used to calibrate the Bayesian model so as to fully capture the features of the parameter set $\boldsymbol{\beta}$ in the linked function. In the following section, the application results at a few selected calibration gauge sites are provided to reflect the operational performance of the proposed Bayesian approach. One calibration gauge is randomly selected from each elevation category. Figure 11 shows the hourly rainfall estimates and rainfall accumulations at four selected calibration gauge stations. These four calibration sites are at the height of $29,79,151,518 \mathrm{~m}$, respectively, covering the four elevation categories. Figure 11 also shows that the bias corrected radar QPE products are dramatically better than those before correction. The bias corrected products agree very well with gauge observations, especially after incorporating the terrain topography into the Bayesian framework. In addition, it is noted that the terrain information has a stronger impact at higher elevations (see results at site "PVW").

Figure 12 shows the scatterplots of hourly rainfall estimates at all 27 calibration gauge stations. Their PDFs are illustrated in Fig. 13. It can be seen from Fig. 12 that the bias corrected radar QPE products agree very well with calibration gauge observations, and the $95 \%$ confidence intervals are very narrow, demonstrating the robustness of the Bayesian correction technique. In particular, the PDF of bias corrected radar QPE after integrating the terrain information is almost identical to the distribution of rain gauge measurements (see Fig. 13).

The quantitative evaluation results of bias corrected hourly rainfall estimates at the 27 calibration gauge sites are included in Table 1 . As expected, the overall
TABLE 1. Evaluation results of hourly rainfall estimates for the 7 Feb 2017 event. The products include radar-only, as well as the corrected estimates using the proposed Bayesian technique with and without the terrain information. Columns (from left to right) refer to the product type, root-mean-square error (RMSE; mm), normalized mean error (NME; \%), normalized mean absolution error (NMAE; \%), and correlation coefficient (CORR).

\begin{tabular}{ccccc}
\hline \hline Rainfall product & RMSE & NME & NMAE & CORR \\
\hline \multicolumn{5}{c}{ At calibration gauge sites } \\
Radar only & 4.66 & -65.3 & 67.4 & 0.36 \\
Bayesian correction & 2.20 & 0.1 & 29.7 & 0.72 \\
$\begin{array}{c}\text { Bayesian correction } \\
\text { (terrain included) }\end{array}$ & 1.90 & 1.4 & 26.4 & 0.80 \\
\multicolumn{5}{c}{} \\
At validation gauge sites \\
Radar only & 4.20 & -60.7 & 64.5 & 0.11 \\
Bayesian correction & 1.71 & 2.0 & 24.2 & 0.74 \\
Bayesian correction & 1.59 & 1.2 & 22.5 & 0.79 \\
(terrain included) & & & & \\
\hline
\end{tabular}

performance after bias correction is similar to that at validation gauge sites. Compared to the radar-only products, the Bayesian corrected results both before and after considering the terrain information show tremendous improvement. After including the terrain information in the Bayesian framework, RMSE improves from 2.20 to $1.90 \mathrm{~mm}$; NMAE: improves from $29.7 \%$ to $26.4 \%$; and CORR improves from 0.72 to 0.80 .

In addition, it should be noted that although rain gauge data for the same precipitation event are used to calibrate the model in this demonstration analysis, it does not mean the proposed Bayesian correction approach would rely on real-time gauge observations or even the gauge observations from the same event. As mentioned, the designed Bayesian framework is very flexible, and can be calibrated using any historical observations for ease of real-time application. As long as the Bayesian correction model is calibrated (i.e., uncertainty parameters are estimated), it can be applied to correct any new radar QPE estimates. Spatially, the application is not limited to the gauge locations used for calibration. Temporally, the application is not limited to the time frames used in the calibration process. The Bayesian correction model can also be updated easily with newer gauge observations. Application errors induced by calibration using observations from different precipitation types will be investigated in future research.

\section{Summary and future work}

Quantitative precipitation estimation (QPE) using operational WSR-88D over complex terrain in the western United States remains a formidable challenge due to the mountainous topography and fundamental coverage gaps of WSR-88D. The limitations of radar 
(a) Gauge "BTO": Elv. $\leq 50 \mathrm{~m}$

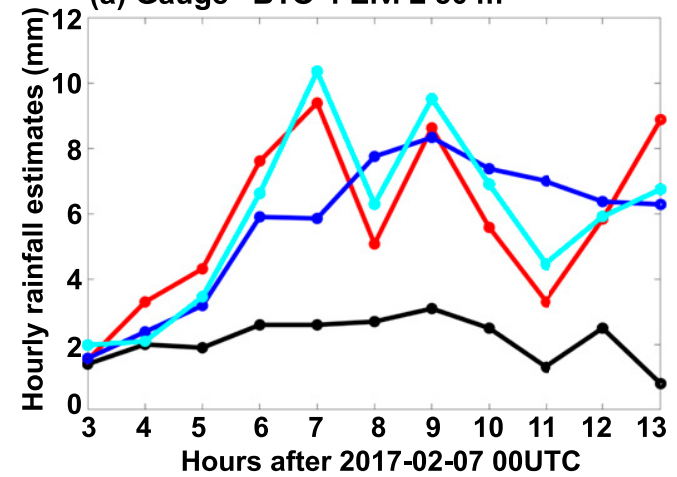

(b) Gauge "GYM": $50 \mathrm{~m}<$ Elv. $\leq 100 \mathrm{~m}$

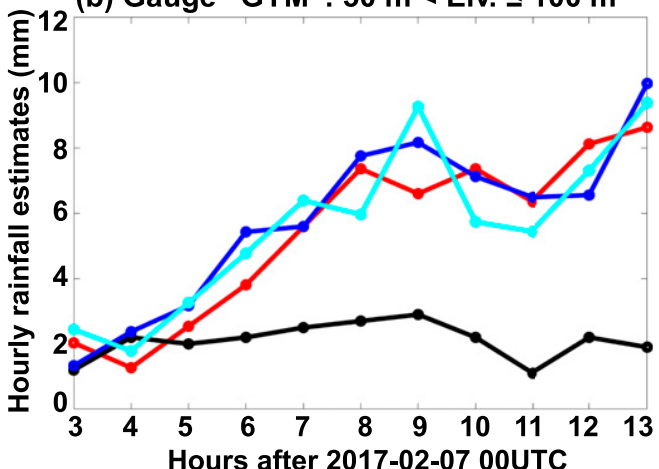

(c) Gauge "KLG": $100 \mathrm{~m}<$ Elv. $\leq 300 \mathrm{~m}$

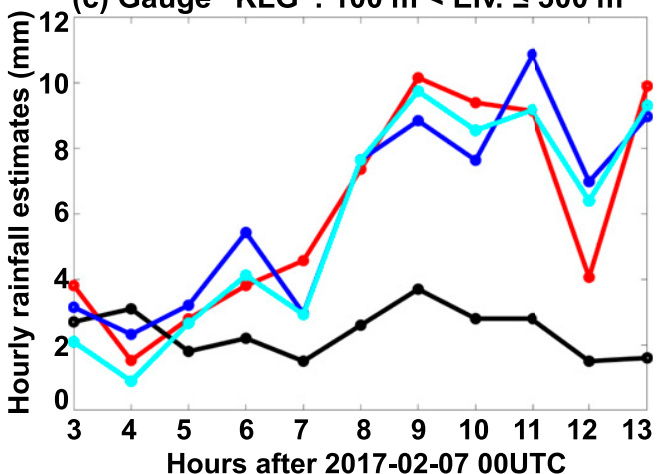

(d) Gauge "PVW": Elv. > $300 \mathrm{~m}$

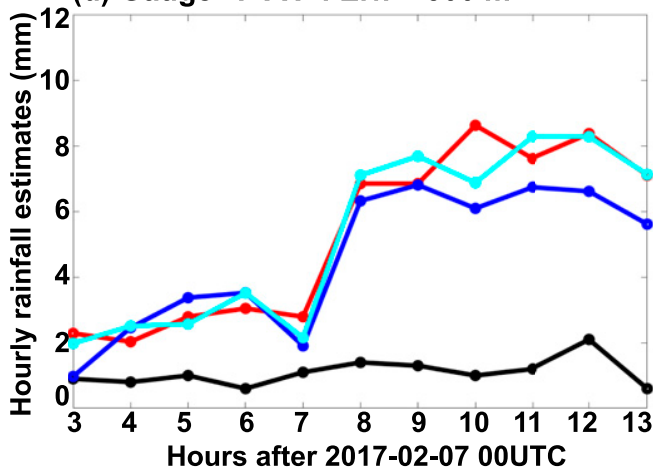

FIG. 11. Hourly rainfall estimates and rainfall accumulations at four selected calibration gauge locations during intense rain hours of the February 2017 precipitation event. Estimates at other calibration gauge locations are not shown since they show similar performance.
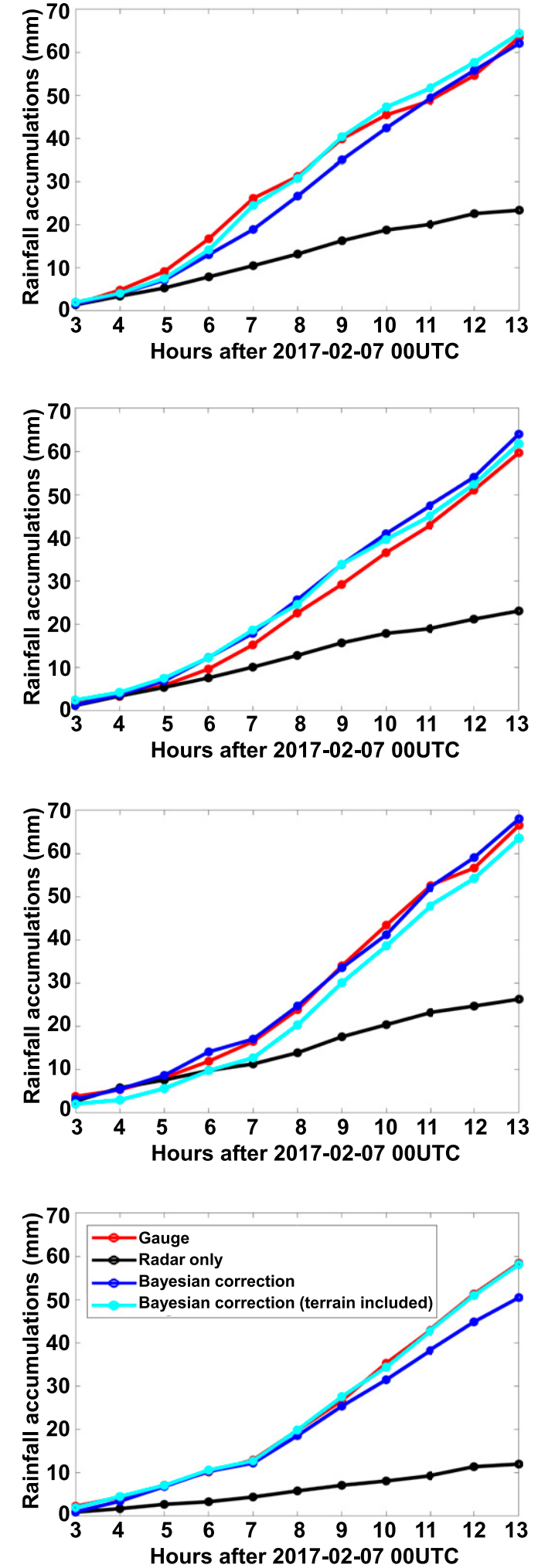


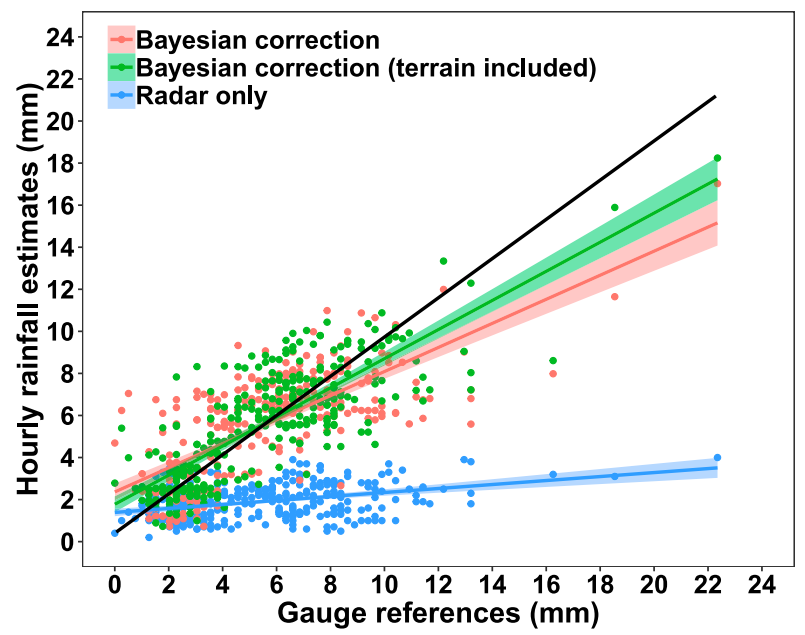

FIG. 12. Scatterplot of hourly rainfall estimates from radar vs calibration gauge observations during the February 2017 precipitation event. The calibration gauge locations are indicated by black dots in Fig. 2. The black solid line represents the 1:1 line, whereas other lines indicate the linear fitting of radar estimates, and the shading areas stand for corresponding $95 \%$ confidence intervals.

sampling geometry are further compounded by the complex precipitation microphysics as a result of orographic enhancement in the mountainous regions. Unfortunately, a number of river basins in the western U.S., including the Northern California region, are in flood-prone areas with poor radar coverage. Current operational radar QPE products are not sufficient to accurately capture the precipitation variations in such domains. Therefore, there is an urgent need to improve operational radar QPE to facilitate our understanding of local stream hydrology and improve flood forecasting.

As part of our continuing effort to improve radar QPE over complex terrain, this study developed a Bayesian framework based on local rain gauge observations to correct the biases and reduce the uncertainties associated with operational radar rainfall products. A demonstration study in the Russian River basin in Northern California is detailed. In addition, conventional local bias and mean field bias correction approaches are implemented for comparison. Primary conclusions are summarized as follows:

1) The proposed Bayesian framework is designed with high flexibility. In addition to radar rainfall intensities, it is capable of incorporating additional factors such as terrain topography and wind information. This Bayesian-based correction technique is also not limited to rainfall estimates at hourly scale. It can be easily extended to other regions and to different temporal scales.

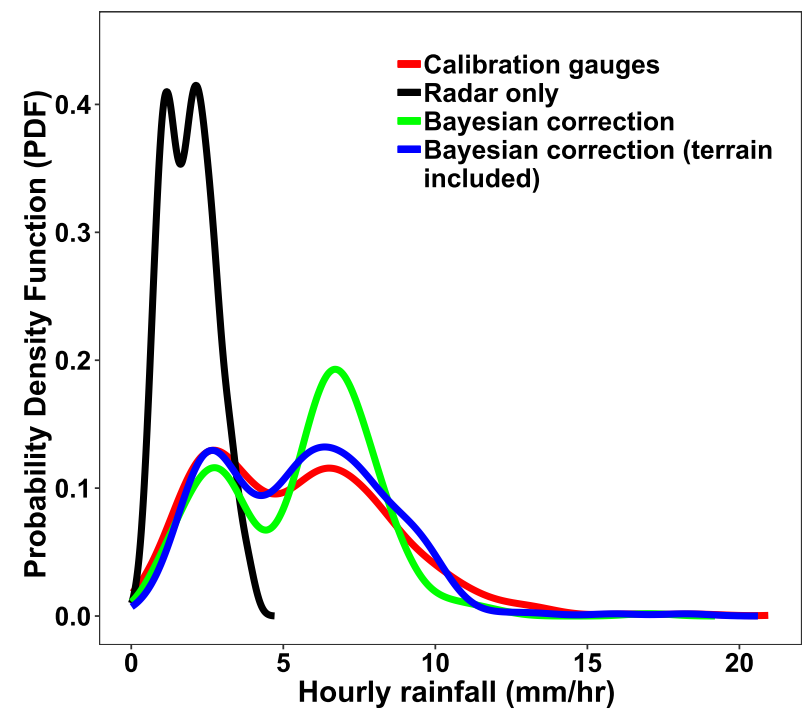

FIG. 13. PDFs of hourly rainfall estimates from radar and calibration gauges shown in Fig. 12.

2) Both the Bayesian correction technique and conventional MFB and LB correction approaches can improve the radar QPE performance.

3) The LB correction performs better than the MFB correction. Based on the validation gauge observations, the RMSEs of MFB and LB corrected results are 2.99 and $2.48 \mathrm{~mm}$, respectively; the NMAEs are $41.0 \%$ and $35.0 \%$, respectively; and the CORRs are 0.11 and 0.53 , respectively.

4) The proposed Bayesian technique performs much better than conventional LB and MFB correction approaches. If only the rainfall intensities are considered in the Bayesian framework, the RMSE, NMAE, and CORR of the corrected QPE are $1.71 \mathrm{~mm}, 24.2 \%$, and 0.74 , respectively. These scores show improvement over previous studies on WSR-88D-based QPE in this complex terrain (e.g., Matrosov et al. 2014; Willie et al. 2017).

5) After incorporating the terrain elevation information in the Bayesian framework, the radar QPE bias correction performance is further enhanced. The RMSE improves from 1.71 to $1.59 \mathrm{~mm}$; NMAE improves from $24.2 \%$ to $22.5 \%$; and CORR improves from 0.74 to 0.79 . Compared to the radar-only products (RMSE: $4.2 \mathrm{~mm}$; NMAE: $64.5 \%$; CORR: 0.11 ), the improvement is dramatic. In fact, these scores are among the best QPE results ever reported for this particular domain. Overall, the QPE performance after including the terrain information is improved about $10 \%$ relatively to that before including the terrain information (i.e., only rainfall intensity values are considered). 
6) In real (operational) applications, real-time gauge data are not required by the proposed Bayesian framework since it can be calibrated using historical observations. It is also suggested that all the reliable gauge stations should be used in the model calibration in order to fully capture the uncertainty distributions.

7) Sample results at calibration gauge sites are presented to reveal the operational performance of the proposed Bayesian approach. The probabilistic distribution curves of bias corrected radar QPE at these sites are almost identical to collocated rain gauge observations, which is very encouraging.

However, it should be noted that the proposed Bayesian model is currently designed conditional on the precipitation events. In reality, rainfall is characterized by large spatiotemporal differences and there may be many zerovalue records. In terms of the probability distribution of rainfall intensity, a zero-inflated model is expected to further improve the Bayesian correction technique, which should be investigated in future. In addition, large scale demonstration prior to possible operational implementation by the MRMS system should be conducted.

Acknowledgments. The authors are grateful to Dr. Paul J. Neiman (NOAA) for numerous discussions on this research. We would also like to thank Dr. Janice Bytheway (NOAA and CU/CIRES) and three anonymous external reviewers for their careful review and comments on this paper. Funding for this research is provided by the California Department of Water Resources, the Sonoma County Water Agency, and the Physical Sciences Division of NOAA Earth System Research Laboratory.

\section{REFERENCES}

Anagnostou, E. N., W. F. Krajewski, and J. Smith, 1999: Uncertainty quantification of mean-areal radar-rainfall estimates. J. Atmos. Oceanic Technol., 16, 206-215, https://doi.org/ 10.1175/1520-0426(1999)016<0206:UQOMAR>2.0.CO;2.

Bellon, A., G. W. Lee, and I. Zawadzki, 2005: Error statistics of VPR corrections in stratiform precipitation. J. Appl. Meteor., 44, 998-1015, https://doi.org/10.1175/JAM2253.1.

Benjamin, S. G., and Coauthors, 2016: A North American hourly assimilation and model forecast cycle: The Rapid Refresh. Mon. Wea. Rev., 144, 1669-1694, https://doi.org/10.1175/ MWR-D-15-0242.1.

Brandes, E. A., 1975: Optimizing rainfall estimates with the aid of radar. J. Appl. Meteor., 14, 1339-134, https://doi.org/10.1175/ 1520-0450(1975)014<1339:OREWTA > 2.0.CO;2.

Bringi, V. N., and V. Chandrasekar, 2001: Polarimetric Doppler Weather Radar: Principles and Applications. Cambridge University Press, 664 pp.

Bytheway, J. L., M. Hughes, K. Mahoney, and R. Cifelli, 2019: A multiscale evaluation of multisensor quantitative precipitation estimates in the Russian River basin. J. Hydrometeor., 20, 447-466, https://doi.org/10.1175/JHM-D-18-0142.1.
Carpenter, B., and Coauthors, 2017: Stan: A probabilistic programming language. J. Stat. Software, 76, 1-32, https://doi.org/ 10.18637/jss.v076.i01.

Chen, H., V. Chandrasekar, and R. Bechini, 2017: An improved dual-polarization radar rainfall algorithm (DROPS2.0): Application in NASA IFloodS field campaign. J. Hydrometeor., 18, 917-937, https://doi.org/10.1175/JHM-D-16-0124.1.

,,- H. Tan, and R. Cifelli, 2019a: Rainfall estimation from ground radar and TRMM Precipitation Radar using hybrid deep neural networks. Geophys. Res. Lett., 46, 10 669-10 678, https://doi.org/10.1029/2019GL084771.

_, R. Cifelli, and A. White, 2019b: Improving operational radar rainfall estimates using profiler observations over complex terrain in Northern California. IEEE Trans. Geosci. Remote Sens., https://doi.org/10.1109/TGRS.2019.2949214, in press.

Cho, H., K. P. Bowman, and G. R. North, 2004: A comparison of Gamma and Lognormal distributions for characterizing satellite rain rates from the Tropical Rainfall Measuring Mission. J. Appl. Meteor., 43, 1586-1597, https://doi.org/ 10.1175/JAM2165.1.

Chumchean, S., A. Sharma, and A. Seed, 2006: An integrated approach to error correction for real-time radar-rainfall estimation. J. Atmos. Oceanic Technol., 23, 67-79, https://doi.org/ 10.1175/JTECH1832.1.

Cifelli, R., V. Chandrasekar, H. Chen, and L. E. Johnson, 2018: High resolution radar quantitative precipitation estimation in the San Francisco Bay Area: Rainfall monitoring for the urban environment. J. Meteor. Soc. Japan, 96A, 141-155, https:// doi.org/10.2151/jmsj.2018-016.

Dettinger, M. D., 2011: Climate change, atmospheric rivers and floods in California-A multimodel analysis of storm frequency and magnitude changes. J. Amer. Water Resour. Assoc., 47, 514 523, https://doi.org/10.1111/j.1752-1688.2011.00546.x.

Doviak, R. J., and D. S. Zrnić, 1993: Doppler Weather Radar and Observations. 2nd ed. Academic Press, 562 pp.

Fulton, R. A., J. P. Breidenbach, D. Seo, D. A. Miller, and T. O'Bannon, 1998: The WSR-88D rainfall algorithm. Wea. Forecasting, 13, 377-395, https://doi.org/10.1175/1520-0434(1998) $013<0377:$ TWRA $>2.0 . \mathrm{CO} ; 2$.

Gou, Y., Y. Ma, H. Chen, and Y. Wen, 2018: Radar-derived quantitative precipitation estimation in complex terrain over the eastern Tibetan plateau. Atmos. Res., 203, 286-297, https:// doi.org/10.1016/j.atmosres.2017.12.017.

Houze, R. A., 2012: Orographic effects on precipitating clouds. Rev. Geophys., 50, RG1001, https://doi.org/10.1029/2011RG000365.

Krajewski, W. F., 1987: Cokriging radar-rainfall and rain gage data. J. Geophys. Res., 92, 9571-9580, https://doi.org/10.1029/ JD092iD08p09571.

_ Adv. Water Resour., 25, 1387-1394, https://doi.org/10.1016/ S0309-1708(02)00062-3.

Maddox, R. A., J. Zhang, J. J. Gourley, and K. W. Howard, 2002: Weather radar coverage over the contiguous United States. Wea. Forecasting, 17, 927-934, https://doi.org/10.1175/15200434(2002)017<0927:WRCOTC>2.0.CO;2.

Matrosov, S. Y., F. M. Ralph, P. J. Neiman, and A. B. White, 2014: Quantitative assessment of operational weather radar rainfall estimates over California's northern Sonoma County using HMT-West data. J. Hydrometeor., 15, 393-410, https://doi.org/ 10.1175/JHM-D-13-045.1.

Ralph, F. M., P. J. Neiman, G. A. Wick, S. I. Gutman, M. D. Dettinger, D. R. Cayan, and A. B. White, 2006: Flooding on California's Russian River: Role of atmospheric rivers. Geophys. Res. Lett., 33, L13801, https://doi.org/10.1029/2006GL026689. 
Seo, D. J., 1998: Real-time estimation of rainfall fields using radar rainfall and rain gage data. J. Hydrol., 208, 37-52, https:// doi.org/10.1016/S0022-1694(98)00141-3.

——, and J. P. Breidenbach, 2002: Real-time correction of spatially nonuniform bias in radar rainfall data using rain gauge measurements. J. Hydrometeor., 3, 93-111, https://doi.org/10.1175/1525-7541(2002)003<0093:RTCOSN $>$ 2.0.CO;2.

_ - — , and E. R. Johnsonb, 1999: Real-time estimation of mean field bias in radar rainfall data. J. Hydrol., 223, 131-147, https://doi.org/10.1016/S0022-1694(99)00106-7.

Todini, E., 2001: A Bayesian technique for conditioning radar precipitation estimates to rain-gauge measurements. Hydrol. Earth Syst. Sci., 5, 187-199, https://doi.org/10.5194/ hess-5-187-2001.

White, A. B., P. J. Neiman, F. M. Ralph, D. E. Kingsmill, and P. O. G. Persson, 2003: Coastal orographic rainfall processes observed by radar during the California Land-falling Jets Experiment. J. Hydrometeor., 4, 264-282, https://doi.org/ 10.1175/1525-7541(2003)4<264:CORPOB $>2.0$.CO;2.
_ - and Coauthors, 2013: A twenty-first-century California observing network for monitoring extreme weather events. J. Atmos. Oceanic Technol., 30, 1585-1603, https://doi.org/ 10.1175/JTECH-D-12-00217.1.

_ B. J. Moore, D. J. Gottas, and P. J. Neiman, 2019: Winter storm conditions leading to excessive runoff above California's Oroville Dam during January and February 2017. Bull. Amer. Meteor. Soc., 100, 55-70, https://doi.org/10.1175/BAMS-D-18-0091.1.

Willie, D., H. Chen, V. Chandrasekar, R. Cifelli, C. Campbell, D. Reynolds, S. Matrosov, and Y. Zhang, 2017: Evaluation of multisensory quantitative precipitation estimation in Russian River basin. J. Hydrol. Eng., 22, E5016002, https://doi.org/ 10.1061/(ASCE)HE.1943-5584.0001422.

Zhang, J., and Y. Qi, 2010: A real-time algorithm for the correction of brightband effects in radar-derived QPE. J. Hydrometeor., 11, 1157-1171, https://doi.org/10.1175/2010JHM1201.1.

_ , and Coauthors, 2011: National Mosaic and Multi-Sensor QPE (NMQ) System: Description, results, and future plans. Bull. Amer. Meteor. Soc., 92, 1321-1338, https://doi.org/ 10.1175/2011BAMS-D-11-00047.1. 